\title{
Effect of Pulse Interval on TSV Process using a Picosecond Laser
}

\author{
Dongsig Shin ${ }^{* 1}$, Jeong Suh $^{* 1}$, and Yongkwon Cho ${ }^{* 1}$ \\ ${ }^{* 1}$ Korea Institute of Machinery \& Materials, 104 Sinseongno, Yuseong-gu, Daejeon, Korea 305-343 \\ Email: dsshin@kimm.re.kr
}

\begin{abstract}
The advantage of using lasers for through silicon via (TSV) drilling is that they allow higher flexibility during manufacturing because vacuums, lithography, and masks are not required; furthermore, lasers can be applied to metal and dielectric layers other than silicon. However, conventional nanosecond lasers have disadvantages including that they can cause heat affection around the target area. In contrast, picosecond lasers enable the precise generation of TSVs with a smaller heat affected zone. In this study, a comparison of the thermal effects around laser drilled holes when using a picosecond laser $(8 \mathrm{ps}, 515 \mathrm{~nm})$ with fluence and repetition rate was conducted. Furthermore, the laser drilling mechanism of silicon was examined through a two-temperature model (TTM) approach to resolve problems related to the heat affection that can be caused by the photo-thermal effect. Notably, the lower fluence and repetition rate of the picosecond laser process reduced the experimentally recast layer, surface debris, and melts around the hole better than the high fluence and short pulse interval condition process. These findings suggest that even the picosecond laser has a heat accumulation effect under high fluence and short pulse interval conditions. However, the long pulse interval due to the low repetition rate can eliminate the heat accumulation effect in the TSV process.

DOI:10.2961/jlmn.2012.02.0002
\end{abstract}

Keywords: TSV, Laser, Drilling, Silicon, TTM

NOMENCLATURE

$\mathrm{D}_{0}=$ ambipolar diffusivity $\left(\mathrm{m}^{2} / \mathrm{s}\right)$

$\mathrm{E}_{\mathrm{p}}=\mathrm{P}_{\mathrm{ave}} /$ Rep: pulse energy (J)

$\mathrm{E}_{\mathrm{t}}=\mathrm{E}_{\mathrm{p}} \times \mathrm{N}$ : total input energy $(\mathrm{J})$

$\mathrm{F}=\mathrm{E}_{\mathrm{p}} /$ area of laser beam: fluence $\left(\mathrm{J} / \mathrm{cm}^{2}\right)$

$\mathrm{I}=$ laser intensity $\left(\mathrm{W} / \mathrm{m}^{2}\right)$

$k_{B}=$ Boltzmann constant $(\mathrm{Js})$

$k=$ thermal conductivity $(\mathrm{W} / \mathrm{mK})$

$\mathrm{N}=$ number of pulses

$\mathrm{N}_{\mathrm{c}}=$ carrier number density $\left(/ \mathrm{m}^{3}\right)$

$\mathrm{P}_{\mathrm{ave}}=$ average power $(\mathrm{W})$

$\mathrm{R}=$ reflectivity

Rep. $=$ repetition rate $(\mathrm{Hz})$

$\mathrm{r}=$ beam radius $(\mu \mathrm{m})$

$\mathrm{T}=$ temperature $(\mathrm{K})$

$\mathrm{U}=$ internal energy $(\mathrm{J})$

$\alpha=$ linear absorption coefficient $(/ \mathrm{m})$

$\delta=$ impact ionization coefficient $(/ \mathrm{s})$

$\gamma=$ Auger recombination coefficient $\left(\mathrm{m}^{6} / \mathrm{s}\right)$

$\phi=$ beam diameter $(\mu \mathrm{m})$

$\lambda=$ wavelength $(\mu \mathrm{m})$

$\tau_{\mathrm{i}}=$ pulse interval: $1 /$ Rep. (sec)

$\tau_{\mathrm{p}}=$ pulse duration (sec)

$\tau_{\mathrm{e}-1}=$ electron relaxation time (sec)

Subscripts

$e:$ electron

$l:$ lattice

\section{Introduction}

Through silicon vias (TSVs) are becoming increasingly important in the semiconductor packaging industry because they can provide compact interconnects in 3D stacking applications. Currently, two technologies, laser drilling and deep reactive ion etching (DRIE), are used to generate these vias and while each method has certain benefits, agreement has not yet been reached on which provides the most effective solution [1].

Laser via machining is an enabling technology for front and back end TSV interconnect applications that provides a reduced cost of ownership (CoO) by eliminating the need for expensive photolithography steps, by increasing throughput, and by offering a platform for simplified seed deposition and accelerated electroplating [1]. With its reliable operation and inexpensive maintenance, laser via machining has also been shown to be a safe process for active devices, especially in comparison with the DRIE process, in which the interplay of the mask-etch steps in a narrow closed channel, such as a via, can result in the formation of sharp, regular scallops that give rise to inter-via electrical leakage current [2].

However, several studies have reported that there are restrictions on achieving precision processing due to heat accumulation and the plasma or particle shielding processes that are inherent in the ablation process when using ultrashort pulsed lasers with short pulse intervals [3, 4]. Moreover, very few studies have been performed on the practical application of ultrashort pulsed lasers, especially picosecond lasers, in micro-drilling silicon wafers.

The present paper presents a comparison between a thermal simulation using the two-temperature model (TTM) [4-8] and experimental results. This comparison allows a practical understanding of the precision drilling of silicon wafers using picosecond lasers. 


\section{Methods}

The experimental setup employed for the TSV is shown in Fig. 1. The applied laser source is a picosecond laser (Trumicro 5250; Trumpf, Germany) with $25 \mathrm{~W}$ average power $\left(P_{\text {ave }}\right), 8$ ps pulse duration $\left(\tau_{p}\right)$, and $515 \mathrm{~nm}$ wavelength $(\lambda)$. The laser beam travels through several mirrors and a scanner (Superscan; Raylase, Germany) to a silicon wafer on an $\mathrm{x}, \mathrm{y}$, and $\mathrm{z}$ automated stage that has $\pm 1 \mu \mathrm{m}$ repeatability and a $0.1 \mu \mathrm{m}$ resolution.

The drilling parameters are the average power $\left(P_{\text {ave }}\right)$, repetition rate (Rep.), pulse energy $\left(E_{p}\right)$, and number of pulses $(N)$; these parameters were selected in order to obtain the pulse interval effects for non-thermal ablation on the TSV process under the same total input energy $\left(E_{t}: 5.8\right.$ $\mathrm{mJ})$. Using an optical microscope and TEM analyses, the shapes of the drilled holes were analyzed and the optimal process parameters were investigated in order to improve the hole quality (Tecnai F30S; FEI, USA).

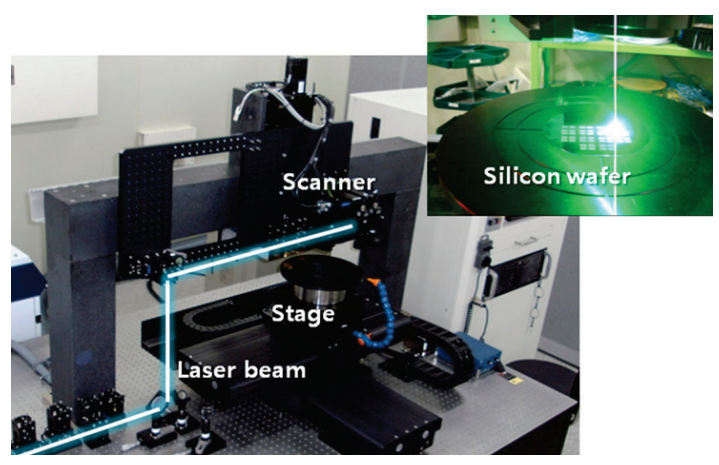

Fig. 1. Experimental setup for the TSV process using a picosecond laser.

Additionally, the investigated results were compared with the simulated temperatures using the TTM $[4,5]$ in order to further increase understanding. The TTM is used extensively to examine the ablation characteristics of a material with single and multi-pulsed ultrashort laser ablations with linear relationships.

Under laser irradiation $(S)$, the present study uses the balance equation of the carrier number density $\left(N_{c}\right)$ to describe the change in the carrier number density that results from the photon energy absorption, as follows:

$$
\begin{gathered}
S(x, y, t)=I_{0} \alpha(1-R) \exp \left(-\left(x-x_{0}\right)^{2} / r^{2}\right) \exp (-\alpha y) \\
\left\{\exp \left(-\left(t-t_{0}\right)^{2} / \tau_{p}^{2}\right)+\exp \left(-\left(t-t_{1}\right)^{2} / \tau_{p}^{2}\right)+\ldots\right\} \\
\frac{\partial N_{c}}{\partial t}=\frac{\partial}{\partial x}\left(D_{0} \frac{\partial N_{c}}{\partial x}\right)+\frac{\partial}{\partial y}\left(D_{0} \frac{\partial N_{c}}{\partial y}\right)-\gamma N_{c}{ }^{3}+\delta\left(T_{e}\right) N_{c}+G(x, y, t)
\end{gathered}
$$

where $D_{0}$ is the ambipolar diffusivity, $\gamma$ is the Auger recombination coefficient, and $\delta\left(T_{e}\right)$ indicates the dependence of the impact ionization coefficient on the electron temperature. $G$ represents the laser absorption source term.

The present study applies the relaxation time approximation to Boltzmann's transport equation and assumes a single thermodynamics system in which the optical phonons and acoustic phonons are in a state of equilibrium.
The electron and lattice energy conservation equations can be described as follows:

$$
\begin{aligned}
& \frac{\partial U_{e}}{\partial t}=\frac{\partial}{\partial x}\left(k_{e} \frac{\partial T_{e}}{\partial x}\right)+\frac{\partial}{\partial y}\left(k_{e} \frac{\partial T_{e}}{\partial y}\right)-\frac{3 N_{c} k_{B}}{\tau_{e-l}}\left(T_{e}-T_{l}\right)+S(x, y, t) \\
& \frac{\partial U_{l}}{\partial t}=\frac{\partial}{\partial x}\left(k_{l} \frac{\partial T_{l}}{\partial x}\right)+\frac{\partial}{\partial y}\left(k_{l} \frac{\partial T_{l}}{\partial y}\right)+\frac{3 N_{c} k_{B}}{\tau_{e-l}}\left(T_{e}-T_{l}\right)
\end{aligned}
$$

where $U_{e}$ and $U_{l}$ are the electron and lattice internal energies, respectively. $\tau_{e-l}$ is the electron-lattice relaxation time, and $S$ is the total laser absorption source term.

\section{Results and discussion}

\subsection{Comparison of via holes fabricated using picose- cond and nanosecond lasers}

Under the ultrashort pulsed laser excitation of semiconductors, high density and high temperature electrons can be excited from the valance band into the conduction band. As a result, lattice instability occurs prior to the significant heating of the phonon subsystem, and this damage is referred to as a non-thermal ablation mechanism [9].

Figure 2 provides the TEM images of the sidewall of a hole drilled using picosecond laser conditions ( $\tau_{p}: 8 \mathrm{ps} ; \lambda$ : $515 \mathrm{~nm} ; E_{p}: 6.5 \mu \mathrm{J}$; Rep.: $\left.200 \mathrm{kHz} ; E_{t}: 5.8 \mathrm{~mJ}\right)$ and nanosecond pulse laser conditions ( $\tau_{p}: 120 \mathrm{~ns} ; \lambda: 355 \mathrm{~nm} ; E_{p}: 60$ $\mu \mathrm{J} ;$ Rep.: $50 \mathrm{kHz} ; E_{t}: 1.2 \mathrm{~mJ}$ ). As shown in Fig. 2, the hole drilled using the picosecond laser lacks the polycrystalline silicon layer that is usually found in a recast layer after thermalization. However, the nanosecond laser drilled hole has a recast layer with a thickness of $2 \mu \mathrm{m}$ even though less total input energy $\left(E_{t}\right)$ was irradiated onto the silicon wafer. The TEM investigations demonstrated that picosecond laser drilling in the range of low fluence $(F)$ results in nonthermal damage to the silicon wafer, while nanosecond laser drilling results in an affected layer on the hole wall of up to $2 \mu \mathrm{m}$ in thickness that contains the amorphous features and polycrystalline silicon; this point is proven by the diffraction pattern shown in Fig. 2(b).

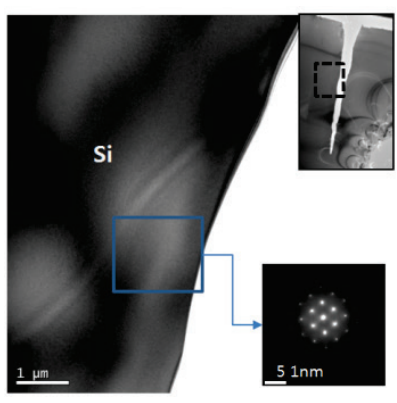

(a)

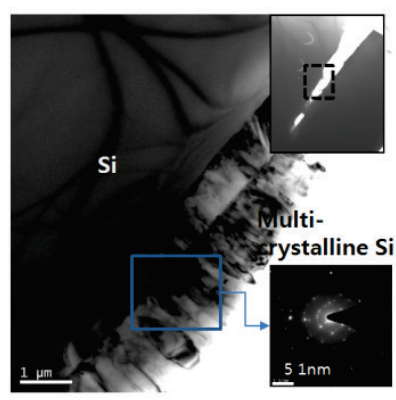

(b)
Fig. 2. TEM images of the hole drilled using (a) picosecond laser $\left(\tau_{p}: 8 \mathrm{ps} ; \lambda: 515 \mathrm{~nm} ; F: 2 \mathrm{~J} / \mathrm{cm}^{2} ; \tau_{i}: 5 \mu \mathrm{s} ; E_{t}: 5.8 \mathrm{~mJ}\right)$ and (b) nanosecond pulse laser processes $\left(\tau_{p}: 120 \mathrm{~ns} ; \lambda: 355 \mathrm{~nm} ; F: 19 \mathrm{~J} / \mathrm{cm}^{2}\right.$; $\left.\tau_{i}: 20 \mu \mathrm{s} ; E_{t}: 1.2 \mathrm{~mJ}\right)$.

3.2 Via drilling according to the fluence of picosecond lasers 
The present study analyzes and minimizes the thermal effects in the holes drilled using a picosecond laser through the use of various parameters. In order to analyze the thermalization of the silicon wafer using the picosecond laser beam, the phenomenon was simulated using the TTM. Figure 3 shows the electron temperature $\left(T_{e}\right)$ and lattice temperature $\left(T_{l}\right)$ with an increase in the fluence $(F)$ under a single pulse $\left(\tau_{p}: 8 \mathrm{ps}\right)$. At an early stage in the laser irradiation, the carrier number density $\left(N_{c}\right)$ increased substantially because the single photon absorption energy creates electrons in the conduction band and holes in the valence band. As time passes, the electron and lattice temperature reach equilibrium through a process of electron-lattice energy relaxations. As shown in Fig. 3, the electron temperature $\left(T_{e}\right)$ sharply increases to $2.4 \times 10^{5} \mathrm{~K}$, whereas the lattice temperature $\left(T_{l}\right)$ is saturated to $1.3 \times 10^{4} \mathrm{~K}$ with increases in the fluence $(F)$. That is, it is difficult to increase the lattice temperature $\left(T_{l}\right)$, which is directly related to the material removal via ablation, even when a higher pulse energy beam is radiated on the material. This indicates that it is not necessary to use a fluence above $6 \mathrm{~J} / \mathrm{cm}^{2}$ in order to obtain effective drilling processes in silicon.

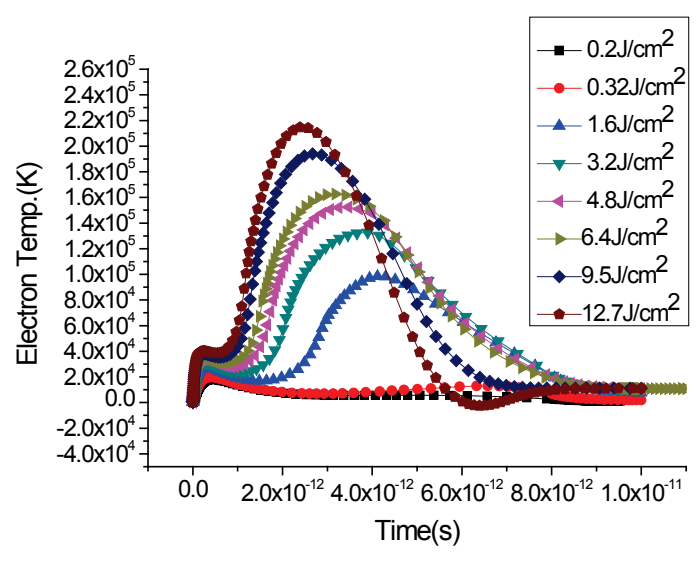

(a)

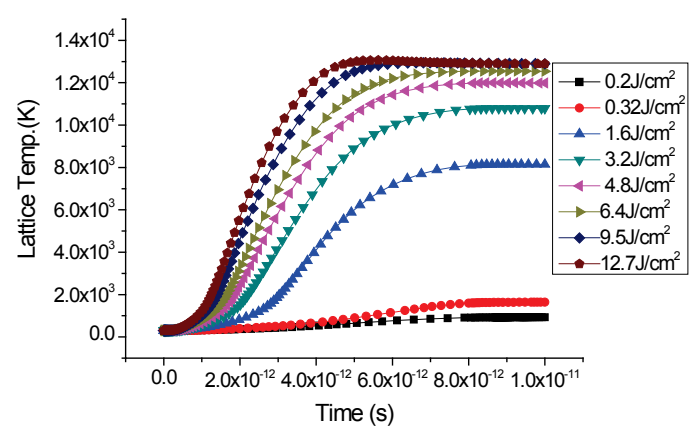

(b)

Fig. 3. Simulated graphs of the (a) electron temperature $\left(T_{e}\right)$ and (b) lattice temperature $\left(T_{l}\right)$ as the fluence $(F)$ and time increase using the TTM.

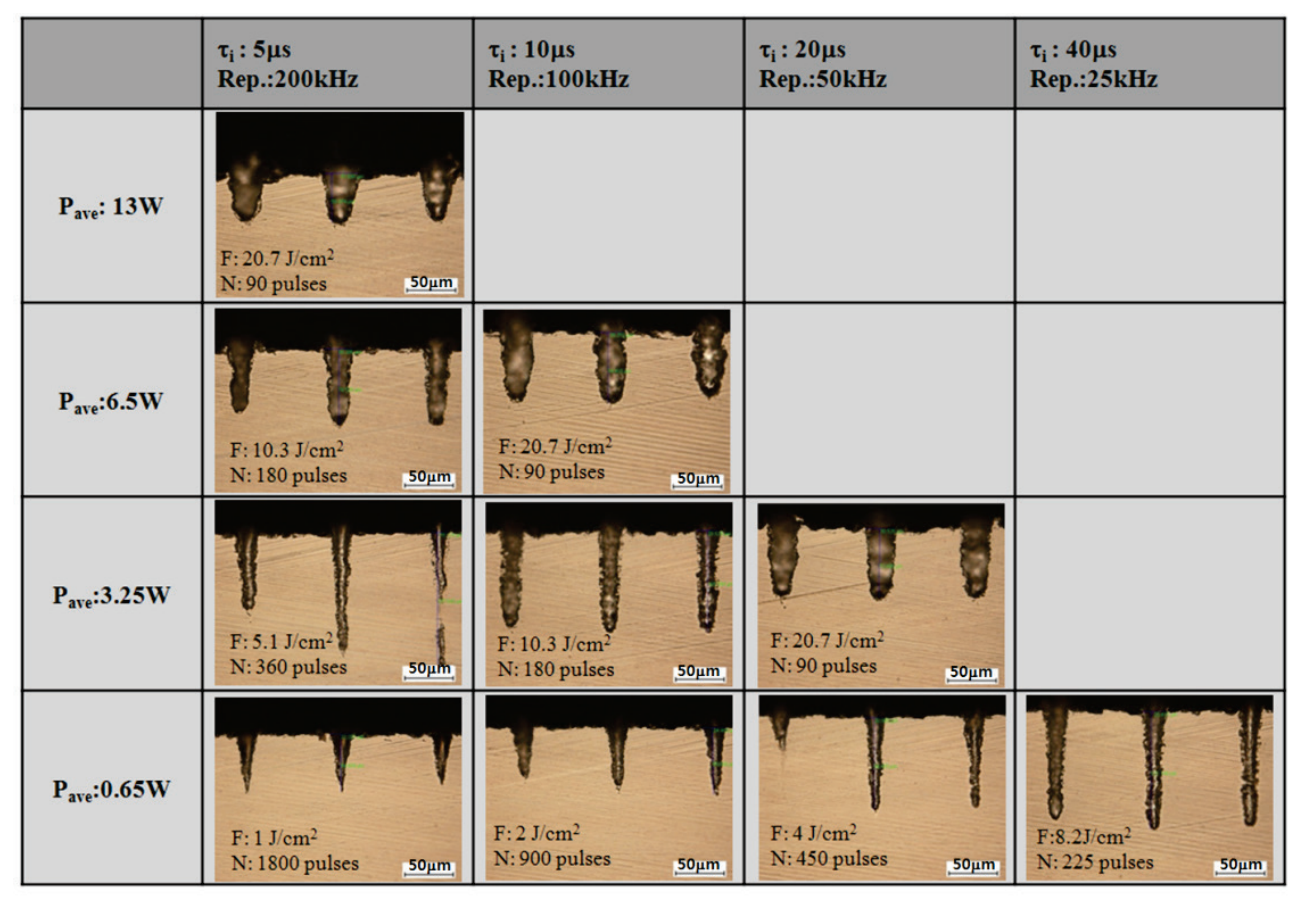

Fig. 4. Cross sectional view of the drilled holes as a function of the average power $\left(P_{\text {ave }}\right)$, fluence $(F)$, and pulse interval $\left(\tau_{i}\right)$ under the same total input energy $\left(E_{t}: 5.8 \mathrm{~mJ}\right)$. 
In order to verify the simulated results, the laser drilled holes were analyzed with an increase of fluence $(F)$. Figure 4 shows a cross sectional view of the drilled holes as a function of the average power $\left(P_{\text {ave }}\right)$, repetition rate (Rep.), and fluence $(F)$ under the same total input energy $\left(E_{t}\right)$ of $5.8 \mathrm{~mJ}$.

A higher average power $\left(P_{\text {ave }}\right)$ is often assumed to be the only method of increasing the productivity of laser processing. However, as shown in Fig. 4, a higher fluence $(F)$ with a high average power generates a wide hole diameter and thermal damage with a higher repetition rate (Rep.). This indicates that the optimum fluence $(F)$ for effective laser drilling cannot exceed $9.5 \mathrm{~J} / \mathrm{cm}^{2}$, as was found in the simulation model in Fig. 3.

Figure 5 shows the graphs of the hole depths and via volumes from the quantitative measurements of the images in Fig. 4. In the graphs in Fig. 5, the hole depth and ablation volume can be classified according to the pulse energy $\left(E_{p}\right)$ and fluence $(F)$. Figure 5(a) shows that the higher pulse energy region (above $F: 8.2 \mathrm{~J} / \mathrm{cm}^{2}$ ) tends to decrease the hole depth. However, the lower fluence region (below $F: 8.2 \mathrm{~J} / \mathrm{cm}^{2}$ ) has different changes than those in the high fluence region: the hole depth grows with increases in the fluence $(F)$.

Figure 5(b) shows graphs of the via volume from quantitative measurements of the images in Fig. 4. In this graph, the via volumes have different transitions compared with the hole according to the pulse energy $\left(E_{p}\right)$ and fluence $(F)$. That is, the entire pulse energy region tends to increase in volume at each average power, while the higher fluence region (above $F: 8.2 \mathrm{~J} / \mathrm{cm}^{2}$ ) tends to decrease the hole depth through rapid explosion and thermalization in the drilled hole. This indicates that the lower fluence region is good for precision drilling (hole diameter: $<10 \mu \mathrm{m}$ ) on silicon wafers, even when using picosecond lasers. Consequently, low power and low pulse energy $\left(E_{p}\right)$ are the preferred conditions for precision machining with deep holes.

\subsection{Comparisons of heat accumulation from the TTM simulation}

Under the ultrashort pulsed laser irradiation on silicon, the diffusive energy is transported using hot electrons during the electron relaxation time $\left(\tau_{e-l}\right)$, beyond which common thermal diffusion dominates and replaces the hot electron diffusion. The coupling between the electrons and phonons is strengthened with the relaxation time and contributes to a higher energy transfer rate from the electrons to the lattice, as stated by $\mathrm{Hu}$ [5]. These results contribute to the energy accumulation between pulses. Similarly, because the initial material boundary for the second laser pulse irradiation has a higher temperature, the thermal effect is increased when compared with the first laser pulse.

In order to clarify and verify this phenomenon, the lattice temperature $\left(T_{l}\right)$ of the material was simulated using the TTM. Figure 6 shows the simulated development of the material temperature to a depth of $1 \mu \mathrm{m}$, with comparisons between short pulse intervals $\left(\tau_{i}\right)$ with high fluence and long pulse intervals with low fluence.

As shown in Fig. 6, the lattice temperature $\left(T_{l}\right)$ increases sharply during the period of laser irradiation, and then decreases in both cases. However, there is a difference in the lattice temperature after the decrease. For the short pulse interval with high fluence laser beam radiation, the base temperature increased gradually to $1022 \mathrm{~K}$ through heat accumulation, whereas the long pulse interval with low fluence laser beam exhibited lattice temperature $\left(T_{l}\right)$ increases only to $511 \mathrm{~K}$ for the fifth pulse. This indicates that the pulse-to-pulse separation time can affect the heat accumulation with a high fluence laser beam.

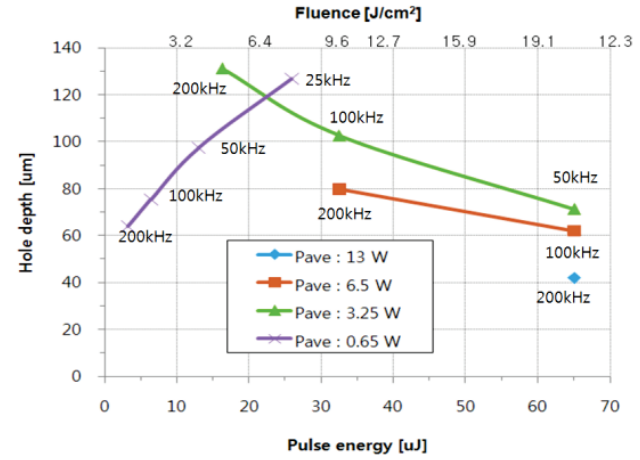

(a)

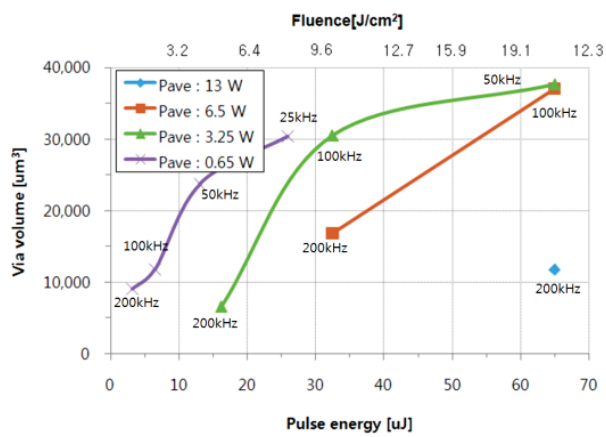

(b)

Fig. 5. Graphs of the (a) hole depth and (b) via volumes as a function of the pulse energy $\left(E_{p}\right)$ and fluence $(F)$ under the same total input energy $\left(E_{t}: 5.8 \mathrm{~mJ}\right)$.

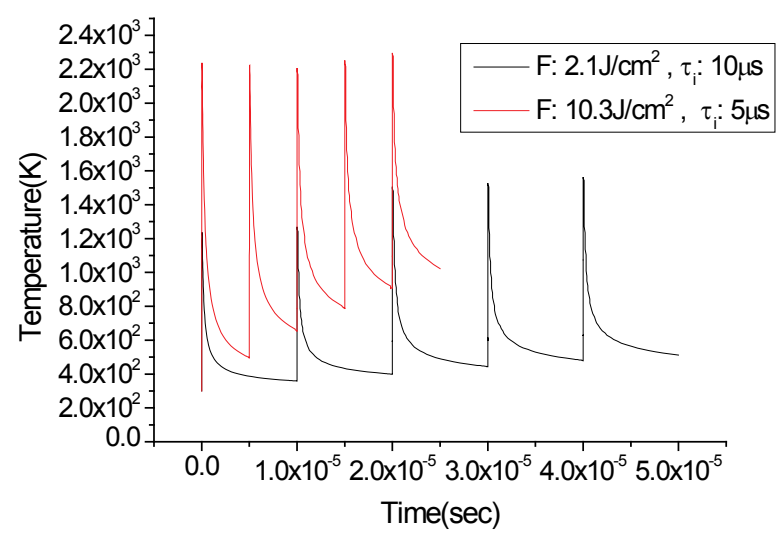

Fig. 6. Simulated lattice temperature $\left(T_{l}\right)$ for comparison of the heat accumulation between the short pulse interval with high fluence $\left(F: 10.3 \mathrm{~J} / \mathrm{cm}^{2} ; \tau_{i}: 5 \mu \mathrm{s}\right)$ and the long pulse interval with low fluence $\left(F: 2.1 \mathrm{~J} / \mathrm{cm}^{2} ; \tau_{i}: 10 \mu \mathrm{s}\right)$ under the same total input energy $\left(E_{t}: 5.8 \mathrm{~mJ}\right)$. 
As illustrated in Fig. 3, the lattice temperature $\left(T_{l}\right)$ of silicon is saturated near $6 \mathrm{~J} / \mathrm{cm}^{2}$ of fluence $(F)$ in the picosecond laser beam radiation. Therefore, a thermal phenomenon was simulated through the heat accumulation between the long and short pulse intervals $\left(\tau_{i}\right)$ near $6 \mathrm{~J} / \mathrm{cm}^{2}$ of fluence $(F)$. As shown in Fig. 6 , there were different lattice temperatures after the decrease as shown in Fig. 7. For the short pulse interval with low fluence laser beam radiation, the base temperature increased gradually to $902 \mathrm{~K}$ through heat accumulation, whereas the long pulse-to-pulse separation time with a higher fluence laser beam increased the lattice temperature $\left(T_{l}\right)$ to only $461 \mathrm{~K}$ in the fifth pulse. This indicates that the pulse interval $\left(\tau_{i}\right)$ dominates the parameters for heat accumulation around the saturation fluence $\left(F: 8.2 \mathrm{~J} / \mathrm{cm}^{2}\right)$. From these results, it can be concluded that precision machining using ultrashort pulsed laser ablation is only available in long pulse intervals. Moreover, long pulse-to-pulse separation times can eliminate the heat accumulation effect that is generated even when using picosecond lasers.

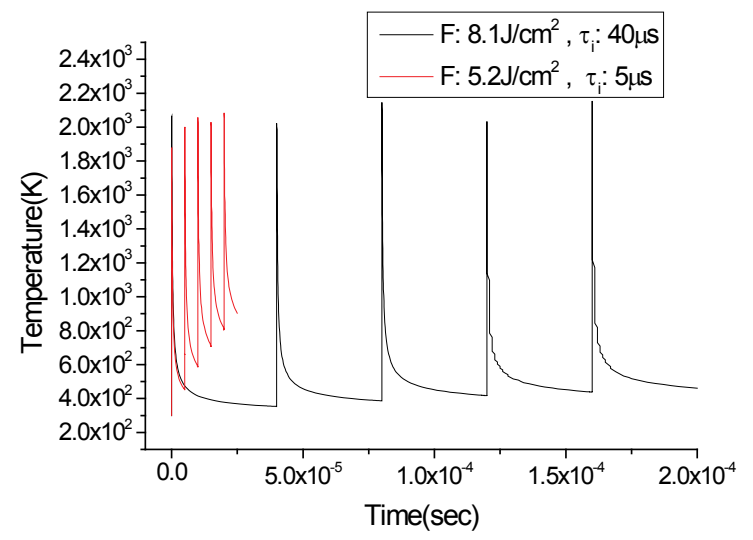

Fig. 7. Simulated lattice temperature $\left(T_{l}\right)$ for comparison of the heat accumulation between short pulse intervals $\left(F: 5.2 \mathrm{~J} / \mathrm{cm}^{2} ; \tau_{i}\right.$ : $5 \mu \mathrm{s})$ and long pulse intervals $\left(F: 8.1 \mathrm{~J} / \mathrm{cm}^{2} ; \tau_{i}: 40 \mu \mathrm{s}\right)$ under the same total input energy $\left(E_{t}: 5.8 \mathrm{~mJ}\right)$.

\subsection{Comparisons of heat accumulation through inves- tigation}

Figure 8 presents the SEM images of a drilled surface under the conditions of a short pulse interval with a high fluence region and a long pulse interval with a lower pulse energy region. As shown in the simulation in Fig. 6, long pulse interval conditions can induce less thermal damage, such as the creation of small holes and melts around the holes, while violent melt ejection was qualitatively observed under short pulse interval conditions even when using the same total input energy, as shown in Fig. 8.

Figure 9 presents the TEM images of the sidewall in a drilled hole using the representative conditions between the short pulse interval with a high fluence region and the long pulse interval with a low fluence region under the same total input energy $\left(E_{t}\right)$. Neither case exhibited evidence of the formation of a polycrystalline layer via thermalization, which is demonstrated by the regular diffraction pattern shown in Fig. 9. However, there is a noteworthy difference between the short pulse interval with a high fluence region and the long pulse interval with a low pulse fluence region.

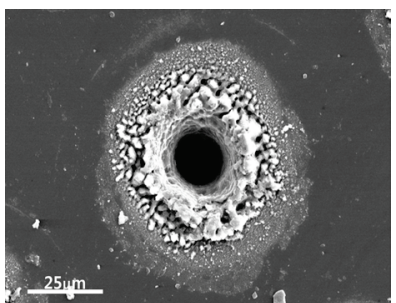

(a)

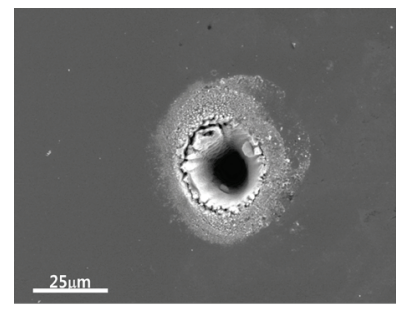

(b)
Fig. 8. SEM images of the drilled surface with the conditions of (a) a short pulse interval with high fluence $\left(F: 10.3 \mathrm{~J} / \mathrm{cm}^{2} ; \tau_{i}: 5\right.$ $\mu \mathrm{s})$ and (b) a long pulse interval with low fluence $\left(F: 2.1 \mathrm{~J} / \mathrm{cm}^{2}\right.$; $\left.\tau_{i}: 10 \mu \mathrm{s}\right)$ under the same total input energy $\left(E_{t}: 5.8 \mathrm{~mJ}\right)$.
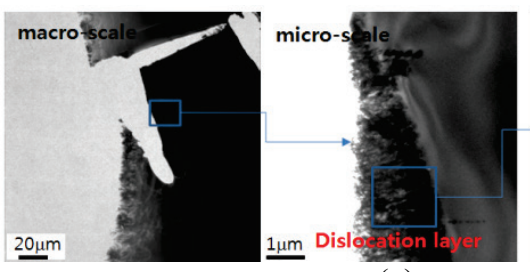

(a)
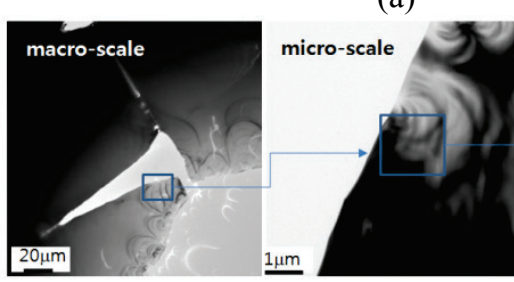

(b)

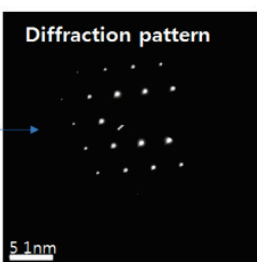

Diffraction pattern

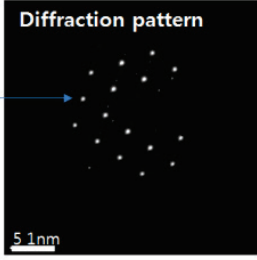

Fig. 9. TEM images and diffraction patterns of the drilled hole with conditions of (a) a short pulse interval with high fluence $(F$ : $\left.10.3 \mathrm{~J} / \mathrm{cm}^{2} ; \tau_{i}: 5 \mu \mathrm{s}\right)$ and (b) a long pulse interval with low fluence $\left(F: 2.1 \mathrm{~J} / \mathrm{cm}^{2} ; \tau_{i}: 10 \mu \mathrm{s}\right)$ under the same total input energy $\left(E_{t}: 5.8\right.$ $\mathrm{mJ})$.

As shown in Fig. 9(a), an irregular, colored dislocation layer, which is characterized by a crystallographic defect, covers the sidewall. This phenomenon is usually generated through mechanical forces. Thus, this implies that the short pulse interval with a higher fluence region, even when using a picosecond laser, can generate mechanical defects during the TSV process. The TEM investigations demonstrate that picosecond laser drilling using a long pulse interval with a low fluence condition is advantageous when avoiding non-thermal damage to the silicon wafer.

\section{Conclusions}

This study provided a method of reducing the thermal effect that occurs when using a nanosecond pulsed lasers based on the TSV drilling process. It was anticipated that the use of a picosecond laser-pulsed drilling process would reduce thermal defects such as melted layers. It was found that the long pulse-to-pulse separation time with lower pulse energy conditions can induce smaller hole sizes and lower melt ejections around the holes while avoiding the thermal defects caused by heat accumulation; whereas violent melt ejection was qualitatively observed under higher fluence conditions even with the picosecond laser. The 
approach outlined in this study can be effective in the precision machining of TSVs for the MCP industry. However, the TSV industry may require higher throughputs for productivity to complement the improved hole quality that can be achieved using picosecond lasers. In order to achieve higher throughputs, a high-speed beam translation system using a short pulse interval of approximately 8.2 $\mathrm{J} / \mathrm{cm}^{2}$ is recommended to obtain less heat accumulation. Details of this system are a subject for future study.

\section{Acknowledgments}

This work was supported by the Korean Ministry of Knowledge Economy as part of the project "MCP Core Technologies for the Next Generation".

\section{References}

[1] A. M. Rodin, J. Callaghan, and N. Brennan: EuroAsia Semiconductor, 30(6), (2008) 11.

[2] R. Nagarajan, K. Prasad, L. Ebin, and B. Narayanan: Sensors and Actuators A: Physical, 139(1-2), (2007) 323.

[3] J. Schille, R. Ebert, U. Loeschner, P. Regenfuss, T. Suess, and H. Exner: "Micro structuring with highly repetitive ultrashort laser pulses" Proc. of LPM 2008.

[4] H. S. Sim, S. H. Lee, and J. S. Lee: J. Mechanical Science and Technology, 21, (2007) 1847.

[5] W. Hu, Y. C. Shin, and G. King: Appl. Phys. A, 98, (2010) 407.

[6] C. Cheng and X. Xu,: Phys. Rev. B, 72, (2005) 165415.

[7] B. Wu and Y.C. Shin: Appl. Surf. Sci., 247, (2007) 4079.

[8] B. H. Christensen, K. Vestentoft, and P. Balling: Appl. Surf. Sci., 253, (2007) 6347.

[9] J. A. Van Vechten, R. Tsu, and F. W. Saris: Physics Letter A., 74, (1979) 422.

(Received: September 27, 2011, Accepted: February 15, 2012) 